PROCEEDINGS OF THE

AMERICAN MATHEMATICAL SOCIETY

Volume 134, Number 3, Pages 825-830

S 0002-9939(05)08179-7

Article electronically published on September 20, 2005

\title{
FIXED POINTS OF COMPACT KAKUTANI MAPS WITH ANTIPODAL BOUNDARY CONDITIONS
}

\author{
DONAL O'REGAN AND JUAN PERÁN \\ (Communicated by Jonathan M. Borwein)
}

\begin{abstract}
We prove a fixed-point result for compact upper semicontinuous compact-convex-valued multifunctions satisfying antipodal boundary conditions on bounded symmetric subsets of a normed space. Two types or antipodal conditions are considered.
\end{abstract}

\section{INTRODUCTION}

Fixed-point theorems for compact upper semicontinuous compact-convex-valued multifunctions (compact Kakutani maps) satisfying antipodal boundary conditions in infinite-dimensional spaces can be found in the works of A. Granas and T.W. Ma. For example see Theorem $4_{\alpha}$ in [6], where the unit ball in a Banach space is considered. A simple consequence of Theorem 9.3 in 8 is the following result:

Theorem 1.1 (T. W. Ma (1972), A. Granas (1959)). Let $M$ be a closed convex symmetric set in a separated locally convex space $E$ and let $F: M \rightrightarrows E$ be a compact Kakutani map satisfying $x \notin \operatorname{co}(F(x) \cup(-F(-x)))$ for all $x \in \partial M$. Then $F$ has at least one fixed point on $M$.

Observe that, since $0 \in \operatorname{co}(F(0) \cup(-F(-0)))$, we find $0 \notin \partial M$. However $0 \in M$ because $M$ is a convex symmetric set, thus it is a neighborhood of the origin. If $F$ has a fixed point on $\partial M$ we are finished. If not, consider the domain in Theorem 9.3 in 8 equal to the interior of $M$. Theorem 1.1 now follows from Theorem 9.3 in 8 if we recall that the interior of a convex symmetric neighborhood of the origin is an open convex symmetric neighborhood of the origin and that the closures of each of these neighborhoods are the same convex set.

Shiow-Yu Chang published the following fixed-point theorem, where the domain is now bounded and balanced, but not necessarily convex, and the antipodal condition is different from that in Theorem 1.1 (see Theorem 7 in [4]).

Theorem 1.2 (S.-Y. Chang (1994)). Let $M$ be a closed bounded symmetric balanced set at 0 in a locally convex space $E$ and let $F: M \rightrightarrows E$ be a compact Kakutani map. If for each $x \in \partial M$ we have $F(x) \cap(-F(-x)) \neq \emptyset$, then $F$ has at least one fixed point.

Received by the editors October 20, 2004.

2000 Mathematics Subject Classification. Primary 47H10, 54H25.

This research was supported in part by Ministerio de Ciencia y Tecnología (Spain), project MTM2004-06652-C03-03. 
The symmetry condition above is superfluous, because balanced sets are always symmetric. However in [4] Chang considers locally convex spaces to be separable. The statement is not true in general for non-separable spaces.

Chang's proof of Theorem 7 in [4 has two mistakes. First he assumes the existence of bounded neighborhoods in a locally convex space. This is not a fatal error, because if $E$ is not a normable space, then if $M$ is bounded, it has empty interior, so 0 is a fixed point $(0 \in \partial M$ implies $F(0) \cap(-F(0)) \neq \emptyset$ and then $0 \in F(0)$, because $F(0)$ is a convex set). Therefore Theorem 1.2 is trivial if $E$ is not a normable space.

Second, the proof of Theorem 7 depends on Theorem 6 (both in [4) whose proof is wrong, because applying Lemma 3 in Theorem 6 (both in in 44) only guarantees the existence of a single-valued antipodal selection $f$ of $\left.F^{V}\right|_{\bar{U}}$ defined on $\partial U$, so it does not guarantee the existence of a single-valued selection defined on $\bar{U}$ and antipodal on $\partial U$. Of course, the antipodal selection has continuous extensions over $\bar{U}$, but it is not proved in 4 that one of these extensions is a selection of $\left.F^{V}\right|_{\bar{U}}$.

It is a well-known fact that the Borsuk's fixed-point theorem remains valid for compact single-valued maps, with bounded closed symmetric domains (not necessarily convex or balanced) in normed spaces, satisfying an antipodal condition like that in Theorem 1.1 (see for instance Theorem 13.4.2 in 7]). In section 3 we shall extend the last-mentioned theorem to the set-valued case: in Theorem 3.2 we assume an antipodal condition like that in Theorem 1.1, but removing convexity, while in Theorem 3.3 an antipodal condition like that in Theorem 1.2 is considered, but we do not assume the domain to be balanced. In section 2 we present some preliminaries, thus making our exposition self-contained.

\section{BACKGROUND MATERIALS}

Multifunctions. Let $X$ be a non-empty set and let $\mathcal{P}(X)$ denote the set of all subsets of $X$. We define a multifunction $F$ from $X$ to an arbitrary set $Y$, written $F: X \rightrightarrows Y$, to be a map $F: \mathcal{P}(X) \rightarrow \mathcal{P}(Y)$ such that $F(A)=\bigcup_{a \in A} F(\{a\})$. We denote $F(\{x\})$ by $F(x)$.

The map $F^{-}: \mathcal{P}(Y) \rightarrow \mathcal{P}(X)$ defined by $F^{-}(B)=\{x \in X: F(x) \cap B \neq \emptyset\}$ is a multifunction, called the inverse multifunction of $F$. It is easily proved that the composition of two multifunctions $F: X \rightrightarrows Y, G: Y \rightrightarrows Z$ is a multifunction, $G \circ F: X \rightrightarrows Z$. As usual, we identify each map $f: X \rightarrow Y$ with the multifunction $A \subset X \rightarrow f(A)$. Obviously, the map usually denoted as $f^{-1}: \mathcal{P}(Y) \rightarrow \mathcal{P}(X)$ coincides with the multifunction $f^{-}$.

The set $\operatorname{Gr}(F)=\{(x, y) \in X \times Y: y \in F(x)\}$ is called the graph of $F$. A point $x \in X$ such that $x \in F(x)$ is a fixed point of the multifunction $F: X \rightrightarrows X$.

Upper semicontinuity. Let $X$ and $Y$ be Hausdorff $\left(T_{2}\right)$ spaces. A multifunction $F: X \rightrightarrows Y$ is called upper semicontinuous if $F^{-}(C)$ is a closed subset of $X$ for each closed subset $C$ of $Y$, while $F$ is called compact if $F(X)$ is a relatively compact subset of $Y$. A compact multifunction has closed graph if and only if it is a compact upper semicontinuous multifunction with compact values (see 6.1.16, 6.3.1 and 6.3.2 in [2]; recall that a compact $T_{2}$-space is a $T_{4}$-space).

Kakutani maps. If $E$ is a locally convex space and $X$ is a Hausdorff space, an upper semicontinuous multifunction $F: X \rightrightarrows E$ with non-empty compact convex values is called a Kakutani map. The multifunction $F$ is said to be finite dimensional 
if $F$ is compact and $F(X)$ is contained in a finite-dimensional linear subspace of $E$. A non-empty set $M \subset E$ is said to be balanced if $\lambda M \subset M$ for every $\lambda \in \mathbb{R}$ with $|\lambda| \leq 1$, and it is symmetric if $M=-M$.

$\epsilon$-approximations. For $\epsilon>0$ and $X$ a metric space, let $B_{\epsilon}^{X}: X \rightrightarrows X$ be the multifunction $B_{\epsilon}^{X}(x)=\left\{x^{\prime} \in X: d\left(x, x^{\prime}\right) \leq \epsilon\right\}$. Note that $B_{\epsilon}^{X}=\left(B_{\epsilon}^{X}\right)^{-}$. If there is no risk of confusion, we will write $B_{\epsilon}$ instead of $B_{\epsilon}^{X}$. For each $\epsilon>0$ and $F: X \rightrightarrows Y$, where $Y$ is another metric space, let $F_{\epsilon}: X \rightrightarrows Y$ be defined by $F_{\epsilon}(x)=B_{\epsilon}^{Y} \circ F \circ B_{\epsilon}^{X}(x)$. An $\epsilon$-approximation of $F: X \rightrightarrows Y$ is a single-valued mapping $f: X \rightarrow Y$ such that $f(x) \in F_{\epsilon}(x)$ for all $x \in X$. We will make use of the following approximation theorem, essentially due to A. Cellina (see [3]).

Theorem 2.1. A compact Kakutani map $F: X \rightrightarrows E$, from a metric space $X$ to a normed space $E$, has a continuous finite-dimensional $\epsilon$-approximation for each $\epsilon>0$.

To see the finite dimensionality of the $\epsilon$-approximation in Theorem 2.1 first apply Lemma 7.8.2 and later Lemma 7.8.3, both in [7].

Absolute neighborhoods retracts. A metrizable space $Y$ is an absolute neighborhood retract ( $A N R$ ) provided each continuous map $f: D \rightarrow Y$, defined on a closed subset $D$ of any metrizable space $X$, is extendable over some neighborhood of $D$ in $X$ to a map into $Y$. An open subset of an ANR is an ANR. Every locally convex space $E$ is an ANR, thus $E \backslash\{0\}$ is an ANR because each point has a neighborhood that is an ANR; see $\S 11$ in 7 . The following homotopy extension theorem was proved by Borsuk in 1937 (see $\$ 11.6 .2$ in [7]).

Theorem 2.2. Let $D$ be a closed subset of a metrizable space $X$ and let $Y$ be an ANR. If $f, g: D \rightarrow Y$ are homotopic maps and $f$ is extendable over $X$, then $g$ is also extendable over $X$.

Borsuk's Theorem. Borsuk's Theorem for antipodal single-valued maps on convex bounded symmetric domains of $\mathbb{R}^{n}$, initially proved on 1933 , was later extended to normed spaces with antipodal conditions like that in Theorem 1.1 (see 13.4.2 in [7]). We will only need the following version due to L. Nirenberg (1974).

Theorem 2.3. Let $M$ be a closed bounded symmetric set in $\mathbb{R}^{n}$ with $0 \in M$ and let $f: M \rightarrow \mathbb{R}^{n}$ be a continuous map such that $f(x)=-f(-x)$ for each $x \in \partial M$. Then $0 \in f(M)$.

We refer the reader to Theorem 1.7.1 in [9]. Note that if $M$ has empty interior, then the boundary condition in Theorem 2.3 implies that $0=f(0) \in f(M)$. In the other case, apply Theorem 1.7.1 in [9] with $X$ equal to the interior of $M$.

\section{MAin RESUlts}

Let $E$ in the sequel be a normed space. For each $B \subset E$ let $\operatorname{cs}(B)$ denote the set

$$
\operatorname{cs}(B)=\left\{t x+(1-t) x^{\prime} \in E: t \in[0,1], x, x^{\prime} \in B\right\} .
$$

For a multifunction $F: B \rightrightarrows E$ define $\operatorname{cs}(F): B \rightrightarrows E$ by $\operatorname{cs}(F)(x)=\operatorname{cs}(F(x))$.

Lemma 3.1. Let $C \subset D$ be closed subsets of $E$ and let $F: D \rightrightarrows E$ be a compact Kakutani map with no fixed points on $C$. Then, for each $\epsilon>0$ there exists $\delta \in(0, \epsilon)$ such that the multifunction $\operatorname{cs}\left(F_{\delta}\right): D \rightrightarrows E$ is fixed-point-free on $C$. 
Proof. Suppose the statement is false, that is, that $\operatorname{cs}\left(F_{1 / n}\right)$ has a fixed point $x_{n} \in C$ for each $n \in \mathbb{N}$. Then there are

$$
x_{n}^{\prime}, x_{n}^{\prime \prime} \in D, \quad y_{n}^{\prime} \in F\left(x_{n}^{\prime}\right), \quad y_{n}^{\prime \prime} \in F\left(x_{n}^{\prime \prime}\right), \quad z_{n}^{\prime}, z_{n}^{\prime \prime} \in E, \quad t_{n} \in[0,1],
$$

such that

$$
\left|x_{n}^{\prime}-x_{n}\right| \leq 1 / n,\left|x_{n}^{\prime \prime}-x_{n}\right| \leq 1 / n,\left|z_{n}^{\prime}-y_{n}^{\prime}\right| \leq 1 / n,\left|z_{n}^{\prime \prime}-y_{n}^{\prime \prime}\right| \leq 1 / n
$$

and $x_{n}=t_{n} z_{n}^{\prime}+\left(1-t_{n}\right) z_{n}^{\prime \prime}$.

There also exists a subsequence $\left(t_{n_{i}}, y_{n_{i}}^{\prime}, y_{n_{i}}^{\prime \prime}\right)$ of $\left(t_{n}, y_{n}^{\prime}, y_{n}^{\prime \prime}\right)$ in the compact set $[0,1] \times \overline{F(C)} \times \overline{F(C)}$ converging to a point $\left(t_{0}, y_{0}^{\prime}, y_{0}^{\prime \prime}\right) \in[0,1] \times \overline{F(C)} \times \overline{F(C)}$. Obviously, $z_{n_{i}}^{\prime} \rightarrow y_{0}^{\prime}, z_{n_{i}}^{\prime \prime} \rightarrow y_{0}^{\prime \prime}$, thus $x_{n_{i}}=t_{n_{i}} z_{n_{i}}^{\prime}+\left(1-t_{n_{i}}\right) z_{n_{i}}^{\prime \prime} \in C$ converges to $x_{0}=t_{0} y_{0}^{\prime}+\left(1-t_{0}\right) y_{0}^{\prime \prime} \in C$ and then $x_{n_{i}}^{\prime} \rightarrow x_{0}, x_{n_{i}}^{\prime \prime} \rightarrow x_{0}$.

Since $\operatorname{Gr}(F)$ is a closed subset of $M \times E$, we find $y_{0}^{\prime}, y_{0}^{\prime \prime} \in F\left(x_{0}\right)$. Therefore since $F$ is convex valued we have

$$
x_{0}=t_{0} y_{0}^{\prime}+\left(1-t_{0}\right) y_{0}^{\prime \prime} \subset \operatorname{cs}\left(F\left(x_{0}\right)\right) \subset F\left(x_{0}\right),
$$

which contradicts the hypothesis of $F$ having no fixed points on $C$.

We say that a closed symmetric subset $M$ of $E$ is simply symmetric if $M \cap L$ has a bounded connected component containing the origin for each finite-dimensional linear subspace $L$ of $E$. Of course, a closed bounded symmetric set containing the origin is simply symmetric.

Theorem 3.2. Let $M \subset E$ be a simply symmetric set and let $F: M \rightrightarrows L$ be a compact Kakutani map satisfying $F(x) \cap(-F(-x)) \neq \emptyset$ for all $x \in \partial M$. Then $F$ has at least one fixed point on $M$.

Proof. Suppose that $F$ has no fixed points on $M$. Now apply Lemma 3.1 with $C=D=M$, and $\delta>0$ chosen such that

$$
0<\delta<\frac{1}{2} \inf \{d(x, F(x)): x \in M\}
$$

implies $\operatorname{cs}\left(F_{\delta}\right): D \rightrightarrows E$ is fixed-point-free on $M$.

Note that $\inf \{d(x, F(x)): x \in M\}=0$ would imply that $F$ has a fixed point, because $\overline{F(M)}$ is a compact set.

By Theorem 2.1. there is a finite-dimensional linear subspace $L$ of $E$ and two compact continuous maps $f: M \rightarrow L$ and $g: \partial M \rightarrow L$ such that

$$
\begin{gathered}
f(x) \in F_{\delta}(x) \text { for all } x \in M, \\
g(x) \in F_{\delta}(x) \cap\left(-F_{\delta}(-x)\right) \text { for all } x \in \partial M .
\end{gathered}
$$

Note that the multifunction $G: \partial M \rightrightarrows E$ defined by $G(x)=F(x) \cap(-F(-x))$ is a compact Kakutani map and that $G_{\delta}(x) \subset F_{\delta}(x) \cap\left(-F_{\delta}(-x)\right)$.

Since

$$
\operatorname{cs}(\{f(x), g(x)\}) \subset \operatorname{cs}\left(F_{\delta}(x)\right)
$$

and $\operatorname{cs}\left(F_{\delta}\right)$ is fixed-point-free (Lemma 3.1), $x \notin \operatorname{cs}\{f(x), g(x)\}$ for $x \in \partial M$. Define $\psi:(\partial M \cap L) \times[0,1] \rightarrow L \backslash\{0\}$ by

$$
\psi(x, t)=x-t g(x)-(1-t) f(x),
$$

and this is a homotopy joining $\psi_{0}(x)=x-f(x)$ and $\psi_{1}(x)=x-g(x)$.

Analogously, since

$$
\operatorname{cs}(\{g(x),-g(-x)\}) \subset \operatorname{cs}\left(F_{\delta}(x)\right)
$$


then $x \notin \operatorname{cs}\{g(x),-g(-x)\}$ for $x \in \partial M$ and the map $\phi:(\partial M \cap L) \times[0,1] \rightarrow L \backslash\{0\}$ defined by

$$
\phi(x, t)=x+\left(\frac{t}{2}-1\right) g(x)+\frac{t}{2} g(-x)
$$

is a homotopy joining $\phi_{0}=\psi_{1}$ and $\phi_{1}(x)=x-\frac{1}{2} g(x)+\frac{1}{2} g(-x)$.

The map $\psi_{0}: \partial M \cap L \rightarrow L \backslash\{0\}$ is extendable to a $h_{0}: M \cap L \rightarrow L \backslash\{0\}$ defined by $h_{0}(x)=x-f(x)$. Thus, by Theorem 2.2, the map $\phi_{1}: \partial M \cap L \rightarrow L \backslash\{0\}$ is extendable to a $h_{1}: M \cap L \rightarrow L \backslash\{0\}$, and note also that

$$
h_{1}(-x)=\phi_{1}(-x)=-\phi_{1}(x)=-h_{1}(x)
$$

for all $x \in \partial M \cap L$. But this contradicts Theorem 2.3, applied to $h_{1}$ on the connected component of $M \cap L$ containing the origin, whose boundary, as a subset of $L$, is contained in $\partial M \cap L$.

Observe that $B \subset \operatorname{cs}(B) \subset \operatorname{co}(B)$ and that $\operatorname{cs}(B)$ is compact whenever $B$ is compact. Also observe that, for $C, D \subset E$ compact convex sets, the $\operatorname{set} \operatorname{cs}(C \cup D)=$ $\operatorname{co}(C \cup D)$ is convex and compact. Recall that the closed convex envelope of a compact subset is not necessarily compact, unless the space is complete.

Theorem 3.3. Let $M \subset E$ be a simply symmetric set and let $F: M \rightrightarrows L$ be a compact Kakutani map such that $x \notin \operatorname{co}(F(x) \cup(-F(-x)))$ for all $x \in \partial M$. Then $F$ has at least one fixed point on $M$.

Proof. Consider the multifunction $H: M \rightrightarrows L$ defined by

$$
H(x)= \begin{cases}\operatorname{co}(F(x) \cup(-F(-x))) & \text { if } x \in \partial M, \\ F(x) & \text { if } x \notin \partial M,\end{cases}
$$

whose values are compact and convex.

We claim that the graph of $H$ is a closed set and $\overline{G(M)}$ is compact set, thus $H$ is a compact Kakutani map. It suffices to show that for every sequence $\left(x_{n}, y_{n}\right) \in$ $\operatorname{Gr}(H)$ there is a subsequence $\left(y_{n_{i}}\right)$ of $\left(y_{n}\right)$ converging to a point $y_{0}$ such that for every cluster point $x_{0}$ of $\left(x_{n_{i}}\right)$ one has $\left(x_{0}, y_{0}\right) \in \operatorname{Gr}(H)$.

If $\left(x_{n}\right)$ has a subsequence formed by points in the interior of $M$, that is an end to the matter, because $F$ is a compact Kakutani map and $\operatorname{Gr}(F) \subset \operatorname{Gr}(H)$.

Assume that $x_{n} \in \partial M$ for all $n$ so there are

$$
t_{n} \in[0,1], \quad y_{n}^{\prime} \in F\left(x_{n}\right), \quad y_{n}^{\prime \prime} \in F\left(-x_{n}\right)
$$

such that $y_{n}=t_{n} y_{n}^{\prime}-\left(1-t_{n}\right) y_{n}^{\prime \prime}$.

The multifunction $F$ being compact guarantees that $\left(t_{n}, y_{n}^{\prime}, y_{n}^{\prime \prime}\right)$ has a subsequence $\left(t_{n_{i}}, y_{n_{i}}^{\prime}, y_{n_{i}}^{\prime \prime}\right)$ converging to $\left(t_{0}, y_{0}^{\prime}, y_{0}^{\prime \prime}\right)$. Then $y_{n_{i}} \rightarrow y_{0}=t_{0} y_{0}^{\prime}-\left(1-t_{0}\right) y_{0}^{\prime \prime}$.

If there is any cluster point $x_{0}$ of $\left(x_{n_{i}}\right)$, then $y_{0}^{\prime} \in F\left(x_{0}\right), y_{0}^{\prime \prime} \in F\left(-x_{0}\right)$ so

$$
y_{0} \in \operatorname{co}\left(F\left(x_{0}\right) \cup\left(-F\left(-x_{0}\right)\right)\right)=H\left(x_{0}\right) \text {. }
$$

We conclude by applying Theorem 3.2 to $H$, because $H(-x)=-H(x)$ for all $x \in \partial M$. Observe that $H$ has no fixed points on $\partial M$ and coincides with $F$ in the interior of $M$; thus a fixed point of $H$ in the interior of $M$ is a fixed point of $F$. 


\section{REFERENCES}

[1] R. P. Agarwal, M. Meehan and D. O'Regan, Fixed point theory and applications, Cambridge Universty Press, Cambridge, 2001. MR.1825411 (2002c:47122)

[2] G. Beer, Topologies on Closed and Closed Convex Sets, Kluwer Academic Publishers, Dordrecht, 1993. MR1269778 (95k:49001)

[3] A. Cellina, A Theorem on the approximation of compact multi-valued mappings, Atti Accad. Naz. Lincei Cl. Sci. Fis., Mat. Natur. Rend. (8) 47 (1969), 429-433. MR0276936 (43:2676)

[4] S. Y. Chang, Borsuk's antipodal and fixed-point theorems for set-valued maps, Proc. Amer. Math. Soc. 121 (1994) no. 3, 937-941. MR.1221720 (94i:55003)

[5] L. Górniewicz, Topological fixed point theory of multivalued mappings, Mathematics and its Applications, No. 495, Kluwer Academic Publishers, Dordrecht, $1999 . \quad$ MR 1748378 (2001h:58010)

[6] A. Granas, Theorem on antipodes and theorems on fixed points for a certain class of multivalued mappings in Banach spaces, Bull. Acad. Polon. Sci. Sér. Sci. Math. Astr. Phys. 7 No. 5 (1959), 271-275. MR0117588 (22:8365)

[7] A. Granas and J. Dugundji, Fixed point theory, Springer Monographs in Mathematics, Springer-Verlag, New York, 2003. MR.1987179 (2004d:58012)

[8] T.W. Ma, Topological degrees of set-valued compact fields in locally convex spaces, Dissertationes Math. Rozprawy Mat. 92 (1972). MR0309103 (46:8214)

[9] L. Nirenberg, Topics in nonlinear functional analysis, New York Univ. Lecture Notes, New York, 1974.

Department of Mathematics, National University of Ireland, Galway, Ireland

Departamento de Matemática Aplicada, Universidad Nacional de Educación a Distancia, Apartado 60149, Madrid, 28080, Spain

E-mail address: jperan@ind.uned.es 